\title{
Quadro clínico-patológico do envenenamento crotálico experimental em bubalinos comparado com o de bovinos ${ }^{1}$
}

\author{
José Diomedes Barbosa ${ }^{2 *}$, Melina Garcia Saraiva de Sousa ${ }^{2}$, Carlos Hubinger Tokarnia ${ }^{3}$, \\ Marilene de Farias Brito ${ }^{4}$, Alessandra dos Santos Belo Reis ${ }^{2}$, Henrique dos Anjos Bomjardim², \\ Cinthia Távora de Albuquerque Lopes ${ }^{2}$ e Carlos Magno Chaves Oliveira ${ }^{2}$
}

\begin{abstract}
Barbosa J.D., Sousa M.G.S., Tokarnia C.H., Brito M.F., Belo Reis A.S., Bomjardim H.A., Lopes C.T.A. \& Oliveira C.M.C. 2011. [Comparison between the clinical and pathological picture in the experimental poisoning by crotalic venom.] Quadro clínico-patológico do envenenamento crotálico experimental em bubalinos comparado com o de bovinos. Pesquisa Veterinária Brasileira 31(11):967-973. Central de Diagnóstico Veterinário, Universidade Federal do Pará, Rua Maximino Porpino da Silva 1000, Pirapora, Castanhal, PA 68743-080, Brazil. E-mail: diomedes@ufpa.br

The objective of the study was to verify the sensibility of buffaloes to the poison of $\mathrm{Cro}$ talus durissus terrificus and to study the clinical-pathological picture in buffaloes in comparison with the one in cattle. The subcutaneous inoculation of the liofilized poison of the snake, diluted in $1 \mathrm{ml}$ of physiologic solution, was done in the area of the humerus-radio-ulnar joint of three buffaloes at doses of $0.015,0.03$ and $0.066 \mathrm{mg} / \mathrm{kg}$, and of two cattle at doses of 0.03 and $0.066 \mathrm{mg} / \mathrm{kg}$. The buffalo that received the $0.03 \mathrm{mg} / \mathrm{kg}$ dose presented severe clinical signs but recovered six days later. The bovine that received the same dose, died after a clinical course of $22 \mathrm{~h} 56 \mathrm{~min}$. The $0.066 \mathrm{mg} / \mathrm{kg}$ dose caused death of the bovine as also the buffalo, with a clinical course of $4 \mathrm{~h} 23 \mathrm{~min}$ and $8 \mathrm{~h} 12 \mathrm{~min}$, respectively. The buffalo that received the $0.015 \mathrm{mg} / \mathrm{kg}$ dose recovered, after a course of 48 hours. The buffalo that died, showed clinical signs from $3 \mathrm{~h} 58 \mathrm{~min}$ on, and the buffaloes that showed symptoms from $17 \mathrm{~h} 25 \mathrm{~min}$ and $24 \mathrm{~h} 00 \mathrm{~min}$ after inoculation of the venom, but recovered. In the two cattle that died (with doses of 0.03 and $0.066 \mathrm{mg} / \mathrm{kg}$ ), the first clinical signs occurred $6 \mathrm{~h} 10 \mathrm{~min}$ and $6 \mathrm{~h} 31 \mathrm{~min}$ after the inoculation of the venom. The inoculation produced in the buffaloes and cattle nervous symptoms of flaccid paralysis. The main signs observed in the buffaloes as well as the cattle, were slight volume increase at the site of inoculation, respiration difficulties characterized by predominantly abdominal breathing, apathy, sialorreia, difficulty to get up when stimulated, evolution to sternal decubitus followed by lateral decubitus with peddling movements of the legs, and decrease of the reflexes related to the cranial nerves. The buffaloes showed also augmentation of the sustentation base, dragging of the hooves of the hind legs, slow and staggering gait, difficulty in apprehension of the food, The cattle showed additionally paralysis of the eyeballs, revealed through non-exhibition of the sclera during head rotation in latero-caudal direction. Laboratory exams revealed in the cattle and the buffaloes leucocytosis by neutrofilia, and in the biochemistry series, increase in the levels of alanine aminotransferase, aspartato aminotransferase, creatinaquinase and lactic dehydrogenase. There was no alteration in the urinalysis nor
\end{abstract}

\footnotetext{
${ }^{1}$ Recebido em 14 de julho de 2011.

Aceito para publicação em 28 de julho de 2011.

${ }^{2}$ Central de Diagnóstico Veterinário (Cedivet), Faculdade de Medicina Veterinária, Universidade Federal do Pará (UFPA), Rua Maximino Porpino da Silva 1000, Castanhal, PA 68740-080, Brasil. *Autor para correspondência: diomedes@ufpa.br
}

\footnotetext{
${ }^{3}$ Departamento de Nutrição e Pastagem, Instituto de Zootecnia, Universidade Federal Rural do Rio de Janeiro (UFRRJ), Seropédica, RJ 23890-000, Brasil.

${ }^{4}$ Departamento de Epidemiologia e Saúde Pública, Instituto de Veterinária, Universidade Federal Rural do Rio de Janeiro, Seropédica, RJ 23890000 .
} 
in the activation time of protrombine and in the time of partially activated tromboplastin. Necropsy only evidenced slight edema corresponding to the inoculation site in one bovine. Histopathological examination revealed picnosis of the epitelial cell nuclei of some kidney tubules in the cortex (in the buffalo and in one bovine) and slight vacuolation of hepatocites (in one bovine).

INDEX TERMS: Crotalic poisoning, Crotalus durissus terrificus, buffaloes, cattle, snakebite accident.

RESUMO.- 0 estudo teve por objetivo verificar a sensibilidade dos bubalinos à peçonha de Crotalus durissus terrificus, estudar o quadro clínico-patológico e laboratorial nessa espécie e estabelecer comparações com o verificado em bovinos. A inoculação do veneno liofilizado de Crotalus durissus terrificus, diluído em $1 \mathrm{ml}$ de solução fisiológica, foi feita na região da articulação úmero-rádio-ulnar, por via subcutânea, em três bubalinos (doses de 0,015; 0,03 ; e $0,066 \mathrm{mg} / \mathrm{kg}$ ) e em dois bovinos (doses de 0,03 e $0,066 \mathrm{mg} / \mathrm{kg}$ ). 0 bubalino que recebeu a dose de $0,03 \mathrm{mg} /$ kg apresentou sinais clínicos graves recuperou-se seis dias após, e o bovino que recebeu a mesma dose morreu com evolução de $22 \mathrm{~h} 56 \mathrm{~min}$. A dose de $0,066 \mathrm{mg} / \mathrm{kg}$ causou a morte tanto do bovino quanto do bubalino, com evolução clínica de $4 \mathrm{~h} 23 \mathrm{~min}$ e $8 \mathrm{~h} 12 \mathrm{~min}$, respectivamente. 0 bubalino que recebeu a dose de $0,015 \mathrm{mg} / \mathrm{kg}$, recuperou-se com evolução de 48 horas. Os sinais clínicos tiveram início dentro de $3 \mathrm{~h} 58 \mathrm{~min}$ no bubalino que morreu, e nos bubalinos que adoeceram mas se recuperaram, dentro de $17 \mathrm{~h} 25 \mathrm{~min}$ e 24h00min após a inoculação do veneno. Nos dois bovinos que morreram (com doses de 0,03 e $0,066 \mathrm{mg} / \mathrm{kg}$ ), os primeiros sinais clínicos foram observados $6 \mathrm{~h} 10 \mathrm{~min}$ e 6h31min após a inoculação do veneno. A inoculação do veneno produziu nos búfalos e bovinos um quadro nervoso de paralisia flácida. Os principais sinais observados tanto nos búfalos quanto nos bovinos, foram discreto aumento de volume no local da inoculação, dificuldade respiratória caracterizada por respiração predominantemente abdominal, apatia, sialorreia, dificuldade para se levantar quando estimulados, evolução para decúbito esternal permanente, seguido de decúbito lateral e movimentos de pedalagem, e diminuição dos reflexos relacionados aos pares de nervos cranianos. Nos bubalinos adicionalmente foi observado aumento da base de sustentação, arrastar das pinças dos membros posteriores, marcha lenta e cambaleante, dificuldade na apreensão dos alimentos; nos bovinos ainda foram observados paralisia do globo ocular, revelada através da não exposição da esclera durante a rotação da cabeça na direção latero-caudal. Tanto nos bovinos quanto nos bubalinos, verificou-se no leucograma, leucocitose por neutrofilia, e na bioquímica sérica, aumento nos níveis de alanina aminotransferase, aspartato aminotransferase, creatinaquinase e dehidrogenase láctica. Não houve alterações na urinálise, nem no tempo de ativação da protrombina e nem no tempo de tromboplastina parcial ativada. À necropsia evidenciou-se apenas discreto edema correspondente ao local da inoculação em um bovino. Os achados histopatológicos observados foram picnose nos núcleos de células epiteliais de alguns túbulos uriníferos no córtex renal (em um búfalo e um bovino) e fígado com leve vacuolização de hepatócitos (em um bovino).
TERMOS DE INDEXAÇÃO: Envenenamento crotálico, Crotalus durissus terrificus, búfalos, bovinos, acidente ofídico.

\section{INTRODUÇÃO}

O homem do campo costuma atribuir aos acidentes ofídicos e à intoxicação por plantas, a maioria das mortes súbitas em bovinos. Alguns autores acreditam que os acidentes ofídicos seriam muito frequentes e determinariam grandes perdas econômicas aos pecuaristas no Brasil. Contudo, Tokarnia \& Peixoto (2006), com dados disponíveis e em experiência própria, acreditam que os acidentes ofídicos fatais em bovinos são bem menos frequentes, isto é, a sua importância vem sendo bastante superestimada.

A espécie de serpente Crotalus durissus terrificus ${ }^{5}$ habita os cerrados do Brasil central, as regiões áridas e semiáridas do Nordeste, os campos e áreas abertas do Sul, Sudeste e Norte (Melgarejo 2009). Na literatura são descritas três principais ações do veneno crotálico: neurotóxica, miotóxica e coagulante (Azevedo-Marques et al. 2009).

Não há relatos de casos naturais de acidentes por Crotalus em bubalinos e em bovinos no Brasil. Dados sobre o quadro clínico-patológico do envenenamento crotálico, baseados na experimentação, só existem em relação aos bovinos (Araújo et al. 1963, Belluomini et al. 1982, Lago 1996, Bicudo 1999, Tokarnia \& Peixoto 2006, Graça et al. 2008). Sabe-se que em bovinos a dose base de $0,03 \mathrm{mg} /$ kg é capaz de causar a morte dos animais e que a dose de $0,015 \mathrm{mg} / \mathrm{kg}$ também causou a morte de alguns dos animais, sendo que o início dos sinais clínicos e o tempo de evolução são inversamente proporcionais à dose de veneno inoculado, ou seja, quanto maior a dose inoculada mais curto o tempo de evolução (Graça et al. 2008).

Portanto, esse estudo tem por objetivo verificar a sensibilidade dos bubalinos à peçonha de $C$. durissus terrificus, estudar o quadro clínico-patológico e laboratorial nessa espécie e estabelecer comparações com o verificado em bovinos.

\section{MATERIAL E MÉTODOS}

0 experimento foi realizado entre fevereiro e abril de 2010 nas instalações da propriedade Kurikaka no município de Castanhal,

\footnotetext{
${ }^{5}$ De acordo com Melgarejo (2010) apud Aragão et al. (2010) e a Sociedade Brasileira de Herpetologia (2010), recentemente houve modificações na nomenclatura de algumas serpentes. Por exemplo, Crotalus durissus passou a denominar-se Caudisona durissa, Bothrops jararaca agora se denomina Bothropoides jararaca, e Bothrops alternatus é agora designada por Rhinocerophis alternatus. Portanto, com o intuito de evitar confusões, manteremos a nomenclatura antiga para termos de comparação com os demais trabalhos já publicados.
} 
Pará. Foram utilizados dois bovinos e quatro bubalinos, mestiços, clinicamente sadios, dois bovinos fêmeas, três machos bubalinos e uma fêmea bubalina, com peso entre 110 e $150 \mathrm{~kg}$. Um dos bubalinos foi utilizado como controle. Os animais foram mantidos em pastos de Brachiaria spp. e água à vontade. A inoculação do veneno liofilizado de Crotalus durissus terrificus, diluído em $1 \mathrm{ml}$ de solução fisiológica, foi feita na região da articulação úmero-rádio-ulnar, por via subcutânea com seringa de insulina, nos bubalinos nas doses 0,015, 0,03 e 0,066mg/kg e nos bovinos nas doses 0,03 e $0,066 \mathrm{mg} / \mathrm{kg}$. 0 animal controle recebeu $1 \mathrm{ml}$ de solução fisiológica no mesmo local e via de inoculação que os demais.

Foram realizados exames clínicos gerais e neurológicos a cada duas horas até a morte ou a recuperação do animal. Foram realizadas colheitas de sangue dos animais por meio da venopunção da jugular para hemograma, bioquímica sérica e coagulograma, imediatamente antes e depois de $2,6,12$, 24 e 48 horas após a inoculação. As amostras de sangue destinadas à bioquímica sérica foram coletadas em frascos que permitiram a formação e retração do coágulo, com exceção daquelas destinadas ao coagulograma, as quais foram colocadas em frascos com anticoagulante protrombina (Doles ${ }^{\circledR}$ ). As amostras destinadas à realização de hemograma foram colhidas em frascos com ácido etilenodiaminotetra-acético (EDTA) a $10 \%$. As amostras de sangue foram conservadas sob refrigeração e enviadas para o Laboratório de Patologia Clínica da Universidade Federal do Pará, para a realização de hemograma e análise bioquímica; as amostras contendo anticoagulante protrombina foram processadas no laboratório Center Lab em Castanhal. As coletas de urina foram realizadas por micção espontânea durante o exame clínico e por punção vesical durante a necropsia.

No hemograma foi avaliada a série vermelha e a série branca. Na bioquímica sérica foram dosados os níveis de ureia, creatinina, cálcio, fósforo, magnésio, aspartato aminotransferase (AST), alanina aminotransferase (ALT), gama glutamiltransferase (GGT), fosfatase alcalina, bilirrubina direta (BD), bilirrubina total (BT), creatinaquinase (CK) e dehidrogenase láctica (DHL). Para realização destes exames foram utilizados kits comerciais (Bioclín ${ }^{\circledR}$, Doles ${ }^{\circledR}$, Cepa $^{\circledR}$ ) e as leituras foram realizadas em espectofotômetro (Bioplus, modelo Bio 2000). No coagulograma foram realizadas as seguintes análises: tempo de ativação da protrom- bina (TAP), tempo de tromboplastina parcial ativada (TTPA), utilizando-se kits comerciais (Laborlab ${ }^{\circledR}$ ) com determinação manual. Para a urinálise foram utilizadas fitas reagentes (Uri Test 11 Inlab).

As necropsias foram realizadas logo após a morte dos animais. Foram colhidos e imediatamente fixados em formol a $10 \%$ fragmentos de diversos órgãos. Os músculos estriados: masseter, língua, cervical, longíssimo dorsal, diafragma, intercostal, bíceps, psoas, semitendinoso, semimembranoso, pele com tecido subcutâneo e músculos do local da inoculação, antes de fixados, eram previamente expostos ao ambiente por duas horas. Os fragmentos foram submetidos ao processamento de rotina para histopatologia, cortados a $5 \mu$ e corados pela hematoxilina e eosina (HE), no Setor de Anatomia Patológica do Projeto Sanidade Animal, Universidade Federal Rural do Rio de Janeiro.

\section{RESULTADOS}

Os principais dados do experimento encontram-se no Quadro 1.

\section{Dose letal e evolução}

O Bubalino 220 que recebeu a dose potencialmente letal para bovinos $(0,03 \mathrm{mg} / \mathrm{kg})$ apresentou sinais clínicos graves e recuperou-se seis dias após, já o bovino que recebeu a mesma dose morreu. A dose de $0,066 \mathrm{mg} / \mathrm{kg}$ causou a morte tanto do bovino quanto do bubalino. 0 bubalino que recebeu a dose de $0,015 \mathrm{mg} / \mathrm{kg}$ recuperou-se, com evolução de 48 horas.

Os sinais clínicos tiveram início 3h58min no bubalino que morreu, nos bubalinos que adoeceram, mas se recuperaram, $17 \mathrm{~h} 25 \mathrm{~min}$ e $24 \mathrm{~h} 00 \mathrm{~min}$, após a inocolução do veneno. Nos dois bovinos que morreram, os primeiros sinais clínicos foram observados 6h10 min e 6h31min após a inoculação do veneno. As primeiras alterações foram observadas $17 \mathrm{~h} 25 \mathrm{~min}$ após a inoculação, no Bubalino 121 que recebeu a dose de $0,066 \mathrm{mg} / \mathrm{kg}$ foram observadas $3 \mathrm{~h} 58 \mathrm{~min}$ após a inoculação e no Bubalino 090 que recebeu a dose de $0,015 \mathrm{mg} / \mathrm{kg}$ as alterações comportamentais foram observadas 24 horas após a inoculação. No Bovino 1, que rece-

Quadro 1. Envenenamento crotálico em bovinos e bubalinos. Dados principais sobre o delineamento experimental e desfecho

\begin{tabular}{|c|c|c|c|c|c|c|c|}
\hline Animal & Peso & Sexo & Dose & $\begin{array}{c}\text { Data e hora da } \\
\text { inoculação }\end{array}$ & $\begin{array}{l}\text { Início dos sinais } \\
\text { clínicos após a } \\
\text { inoculação }\end{array}$ & $\begin{array}{l}\text { Tempo de } \\
\text { evolução }\end{array}$ & $\begin{array}{c}\text { Recuperação ou } \\
\text { morte após a } \\
\text { inoculação }\end{array}$ \\
\hline Bovino 1 & $110 \mathrm{~kg}$ & Fêmea & $0,066 \mathrm{mg} / \mathrm{kg}$ & $\begin{array}{l}28.02 .10 \\
9 \mathrm{~h} 14 \mathrm{~min}\end{array}$ & 6h31min & 4h23min & $\begin{array}{c}\text { Morreu } \\
\text { 10h54min }\end{array}$ \\
\hline Bovino 2 & $110 \mathrm{~kg}$ & Fêmea & $0,03 \mathrm{mg} / \mathrm{kg}$ & $\begin{array}{c}02.03 .10 \\
13 \mathrm{~h} 40 \mathrm{~min}\end{array}$ & $6 \mathrm{~h} 10 \mathrm{~min}$ & $22 \mathrm{~h} 56 \mathrm{~min}$ & $\begin{array}{c}\text { Morreu } \\
\text { 29h06min }\end{array}$ \\
\hline Bubalino 121 & $123 \mathrm{~kg}$ & Macho & $0,066 \mathrm{mg} / \mathrm{kg}$ & $\begin{array}{c}09.04 .10 \\
19 \mathrm{~h} 17 \mathrm{~min}\end{array}$ & 3h58min & $8 \mathrm{~h} 12 \mathrm{~min}$ & $\begin{array}{c}\text { Morreu } \\
\text { 12h10min }\end{array}$ \\
\hline Bubalino 220 & $120 \mathrm{~kg}$ & Macho & $0,03 \mathrm{mg} / \mathrm{kg}$ & $\begin{array}{c}30.03 .10 \\
15 \mathrm{~h} 45 \mathrm{~min}\end{array}$ & $17 \mathrm{~h} 25 \mathrm{~min}$ & $137 \mathrm{~h}$ & $\begin{array}{c}\text { Adoeceu } \\
\text { gravemente e } \\
\text { recuperou-se em } \\
6 \text { dias }\end{array}$ \\
\hline Bubalino 90 & $150 \mathrm{~kg}$ & Macho & $0,015 \mathrm{mg} / \mathrm{kg}$ & $\begin{array}{c}10.04 .10 \\
12 \mathrm{~h}\end{array}$ & $24 \mathrm{~h} 00 \mathrm{~min}$ & $48 \mathrm{~h}$ & $\begin{array}{l}\text { Adoeceu } \\
\text { levemente e } \\
\text { recuperou-se } \\
72 \mathrm{~h} 00 \mathrm{~min}\end{array}$ \\
\hline $\begin{array}{l}\text { Bubalino } \\
\text { Controle }\end{array}$ & $151 \mathrm{~kg}$ & Fêmea & $\begin{array}{l}\text { 1ml solução } \\
\text { fisiológica }\end{array}$ & $\begin{array}{c}09.04 .10 \\
19 \mathrm{~h} 45 \mathrm{~min}\end{array}$ & - & - & Sem sintomas \\
\hline
\end{tabular}


beu a dose de $0,066 \mathrm{mg} / \mathrm{kg}$, as primeiras alterações foram evidentes a partir de 6h31min após a inoculação e no Bovino 2 que recebeu a dose de $0,03 \mathrm{mg} / \mathrm{kg}$ os sinais clínicos tiveram início $6 \mathrm{~h} 10 \mathrm{~min}$ após a inoculação.

\section{Quadro clínico}

Os principais sinais observados tanto nos búfalos quanto bovinos, foram discreto aumento de volume no local da inoculação, dificuldade respiratória caracterizada por respiração predominantemente abdominal, apatia, sialorreia, dificuldade para levantarem-se quando estimulados (Fig.1), evolução para decúbito esternal permanente (Fig.2) seguido de decúbito lateral e movimentos de pedalagem, e diminuição dos reflexos relacionados aos pares de nervos cranianos (Fig.3). Foi observada discreta diminuição da resposta aos estímulos externos evidenciados pela permissão do contato manual durante o exame clínico, no qual os animais mostravam-se mais tranquilos e não relutavam ao ser colocada a corda na cabeça. As mensurações da frequência cardíaca e da temperatura retal mantiveram-se dentro dos parâmetros normais.

Além dos já mencionados, outros sinais foram observados nos bubalinos. 0 Bubalino 220 logo após a inoculação mostrou-se inquieto e com frequência levava a cabeça ao local da inoculação. Todos os bubalinos apresentaram sensibilidade aumentada ao toque do local inoculado. Os Bubalinos 220 e 121 urinavam frequentemente, principalmente durante a realização dos exames clínicos. No Bubalino 90 verificou-se, logo após a inoculação, discreta mioclonia (tre mores musculares) no local da inoculação. Nos Bubalinos 220 e 121 foram observadas alterações posturais caracterizadas pelo aumento da base de sustentação, cabeça baixa, e quando em decúbito esternal, cabeça voltada para o flanco (postura de auto-auscultação), com evolução para decúbito lateral. Foi observado também pescoço estendido para frente com a cabeça apoiada no chão. No Bubalino 220, quando em movimento, verificou-se arrastar das pinças dos membros posteriores, marcha lenta e cambaleante que, com o agravamento do quadro clínico, relutava em caminhar. Os Bubalinos 121 e 90 se apoiavam nas articulações cárpicas, principalmente no momento de realização dos exames clínicos. O Bubalino 90, durante a marcha, cruzava levemente os membros posteriores. No Bubalino 220 foi observado dificuldade em apreender alimentos, movimento de lateralidade da mandíbula quando movimentada (mandíbula frouxa) e dificuldade na apreensão dos alimentos.

Nos bovinos também foram observados outros sinais. No Bovino 1, o discreto aumento de volume no local da inoculação se estendia até a articulação cárpica; este animal também apresentou vasos episclerais levemente ingurgitados e com o decorrer do tempo apresentou mucosas pálidas. 0 Bovino 2 apresentou mucosa conjuntival pálida no último exame antes do óbito e mioclonia (tremores musculares) em diversos músculos. Nos Bovinos 1 e 2 foi verificada paralisia do globo ocular, revelada através da não exposição da esclera durante a rotação da cabeça na direção latero-caudal.

\section{Patologia clínica}

Na avaliação do eritrograma não foram observadas alterações tanto nos búfalos quanto nos bovinos. No leucograma verificou-se leucocitose em todos os animais, caracterizada por neutrofilia e discreto aumento no número de bastões em um dos bubalinos (Bubalino 220).

Na mensuração dos parâmetros bioquímicos séricos, tanto os bovinos quanto os bubalinos apresentaram aumento nos níveis de ALT, AST, CK e DHL. A fosfatase alcalina aumentou nos Bubalinos 121 e 90, e nos Bovinos 1 e 2. 0 Bubalino controle apresentou aumento nos níveis de AST, fosfatase alcalina, CK e DHL. Os dois bubalinos apresentaram discreto aumento dos níveis de ureia que variou entre 10 a $17 \mathrm{mg} / \mathrm{dL}$ no tempo zero e apresentaram valores máximos que variaram entre 49 e $57 \mathrm{mg} / \mathrm{dL}$. Os níveis de creatinina aumentaram discretamente de 0,3 a 0,5 no tempo zero para 0,9 a $1,9 \mathrm{mg} / \mathrm{dL}$ nos bubalinos, contudo manteve-se dentro da normalidade.

Na análise das amostras de urina não revelou alterações em todos os animais.

Não foram verificadas alterações no TAP e TTPA em todos os animais. Nos bubalinos o tempo de coagulação observado no tempo zero variou de $3 \mathrm{~min} 10 \mathrm{seg}$ a $4 \mathrm{~min} 55 \mathrm{seg}$, sendo que 6 horas após a inoculação atingiu o tempo mínimo de $1 \mathrm{~min} 15 \mathrm{seg}$ e máximo de $7 \mathrm{~min} 30 \mathrm{seg}$. Enquanto que nos bovinos, o tempo de coagulação no tempo zero foi de 45 seg a 2 min 10 seg, que decorridas 6 horas após a inoculação variou de $3 \mathrm{~min}$ a $5 \mathrm{~min} 55 \mathrm{seg}$.

\section{Achados de necropsia e histopatológicos}

À necropsia evidenciou-se apenas discreto edema correspondente ao local da inoculação no Bovino 1.

Os achados histopatológicos observados foram picnose nos núcleos de células epiteliais de alguns túbulos uriníferos no córtex renal (Bovino 1 e Bubalino 121) e fígado com vacuolização dos hepatócitos, principalmente nas zonas centrais e intermediárias do lóbulo hepático, em grau leve (Bovino 2).

\section{Quadro clínico}

\section{DISCUSSÃo}

A inoculação do veneno de Crotalis durissus terrificus tanto nos bovinos quanto nos bubalinos produziu um quadro nervoso de paralisia flácida como observado nos demais experimentos realizados com a espécie bovina no Brasil (Araújo et al. 1963, Belluomini 1972, Lago 1996, Lago et al. 2000, Graça et al. 2008).

0 quadro clínico é caracterizado por hipotonia muscular generalizada, produzido pela ação da crotoxina, neurotoxina pré-sináptica que atua nas terminações nervosas motoras, que resulta em bloqueio neuromuscular e consequentes paralisias motora (paralisia flácida) e respiratória (Azevedo-Marques et al. 2009).

O edema quase imperceptível no local da inoculação já foi descrito por Lago et al. (2000) e Graça et al. (2008) em experimento com bovinos e por Amaral et al. (1991) em humanos; provavelmente passaria despercebido ao exame clínico e mesmo à necropsia, um fator que contribui para a dificuldade do diagnóstico clínico. 
Com o agravamento do quadro clínico todos os animais apresentaram dificuldade respiratória caracterizada por dispneia mista, assim como verificado por Graça et al. (2008) em bovinos.

Constatamos a paralisia do globo ocular nos dois bovinos revelada através da não exposição da esclera durante a rotação da cabeça no sentido horário e anti-horário, alteração descrita por Belluomini et al. (1982) e também verificada por Graça et al. (2008). Este achado clínico constitui indicação importante para estabelecimento do diagnóstico e diagnóstico diferencial do envenenamento crotálico em bovinos, contudo nos bubalinos esta alteração não foi verificada.

A via de inoculação subcutânea foi a mesma utilizada por Graça et al. (2008), porém diferente da utilizada por Belluomini (1972), Lago et al. $(2000,2001,2004)$, que afirmam inocular por via intramuscular $(6 \mathrm{~mm})$, contudo se for
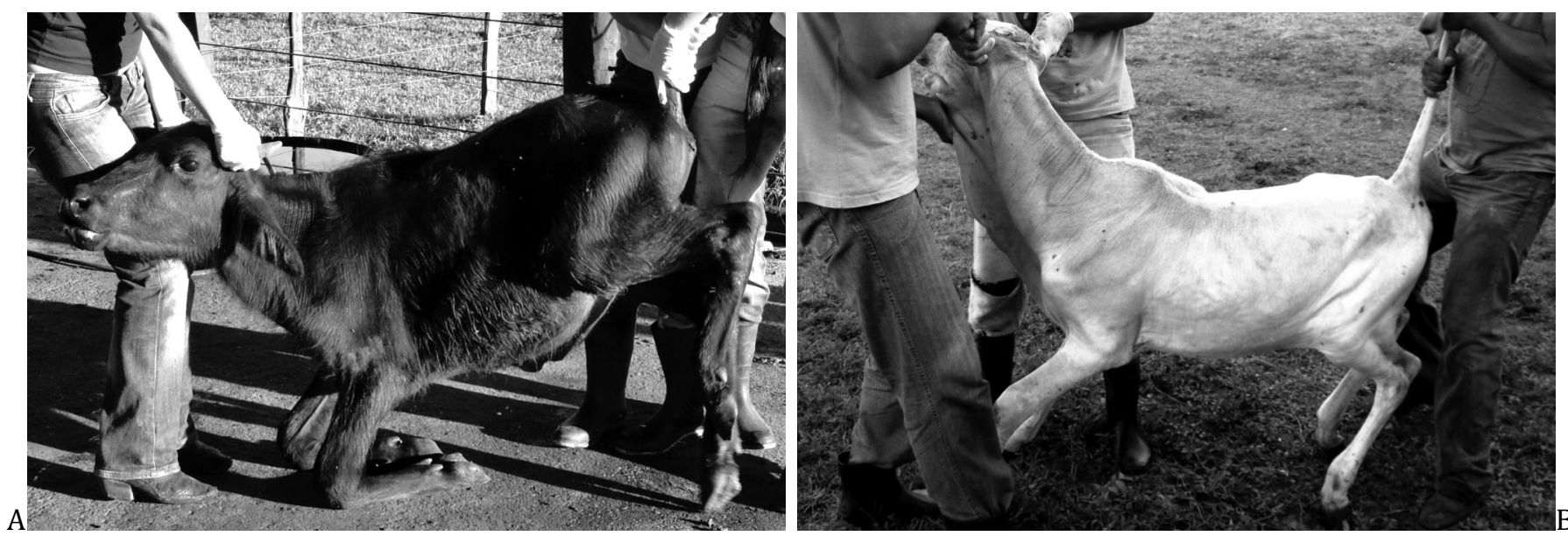

Fig.1. (A) Bubalino 121 e (B) Bovino 1 inoculados com a peçonha de Crotalus durissus terrificus, com incapacidade de se manterem em estação (paralisia flácida).
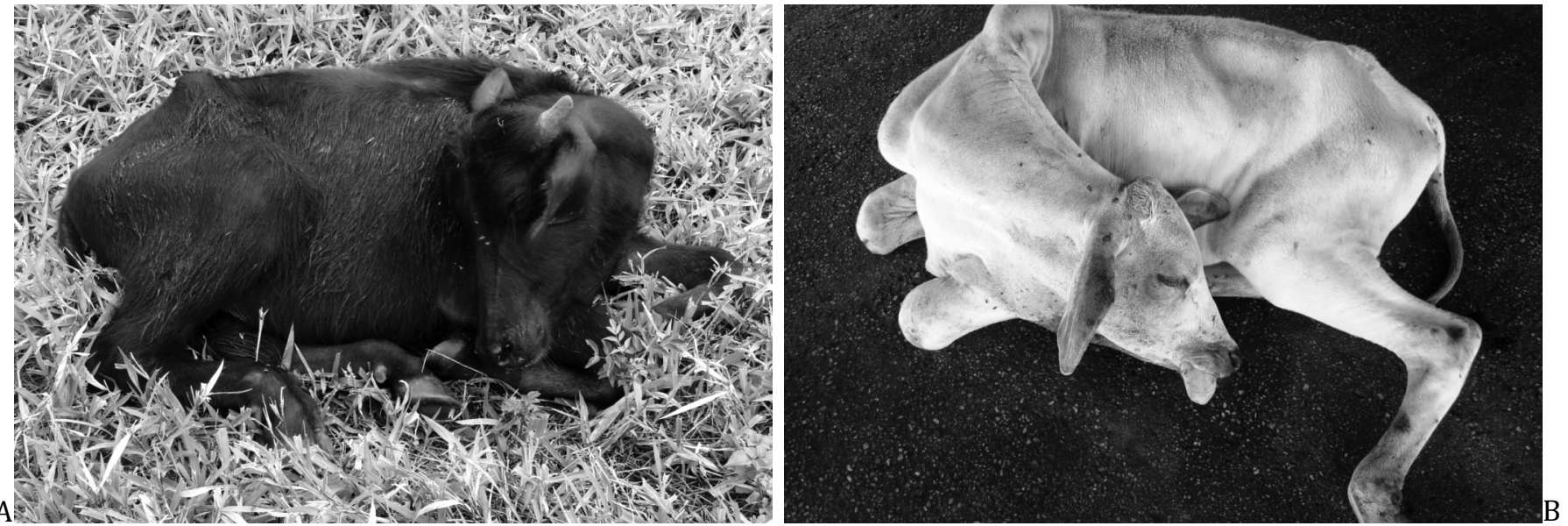

Fig.2. (A) Bubalino 220 e (B) Bovino 2 em decúbito esternal, com postura de auto-auscultação.
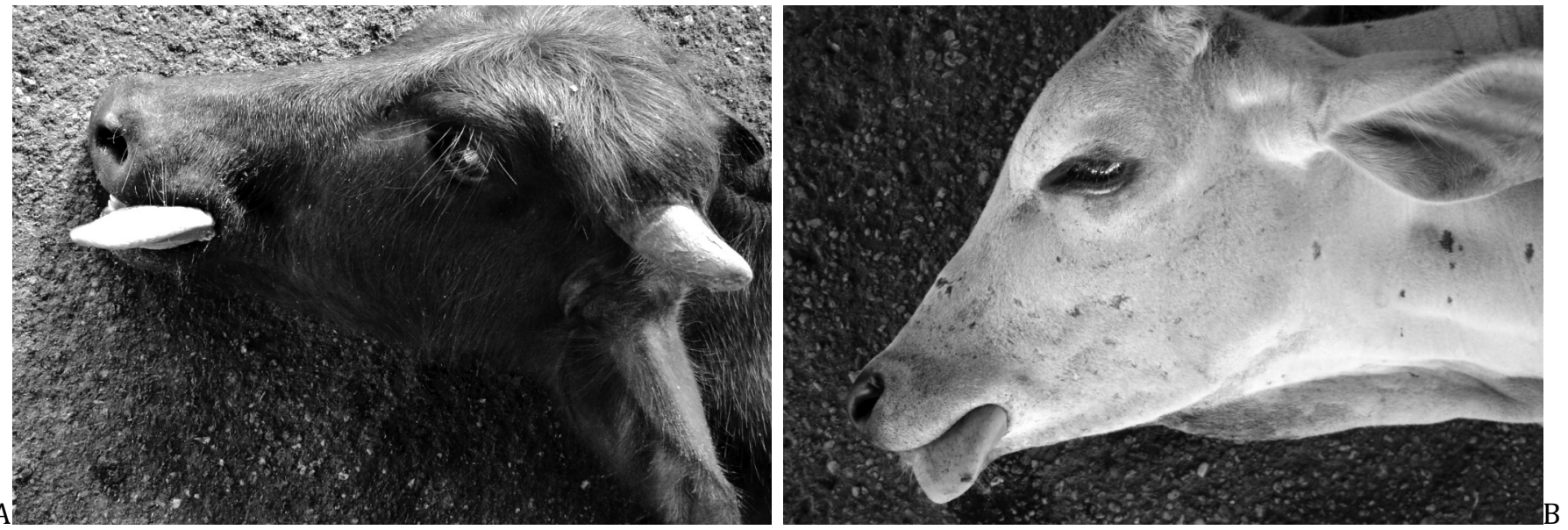

Fig.3. (A) Bubalino 121 e (B) Bovino 2 com diminuição do tônus da língua. 
levado em consideração a espessura da pele de um bovino, que varia entre três e seis milímetros (Sisson 1986), pode-se dizer que esta aplicação foi sobre a fáscia podendo ser caracterizada como subcutânea.

Por tratar-se de um experimento auto-direcionado, no qual os resultados direcionam os próximos experimentos com o objetivo de diminuir ao máximo o número de animais utilizados, optamos pela dose inicial de $0,03 \mathrm{mg} / \mathrm{kg}$, a mesma utilizada por Lago $(1996,2001)$ e Graça et al. (2008) e inferior a aplicada por Araújo et al. (1963).

Como observado também por Graça et al. (2008) em experimentação com bovinos, quanto maior a dose de veneno inoculada mais curto o início do sinais clínicos, com pequena diferença entre o início dos sinais clínicos observada nos bovinos em nosso experimento.

Pode-se verificar que neste experimento os bubalinos foram mais resistentes ao veneno de $C$. durissus terrificus que os bovinos, pois a dose base de $0,03 \mathrm{mg} / \mathrm{kg}$ foi capaz de causar a morte no Bovino 2, mas os Bubalinos 220 e 90 recuperaram-se tanto com a dose de $0,03 \mathrm{mg} / \mathrm{kg}$ quanto com $0,015 \mathrm{mg} / \mathrm{kg}$. Nos experimentos de Graça et al. (2008) a dose de $0,03 \mathrm{mg} / \mathrm{kg}$ causou a morte em um bovino e a dose de $0,015 \mathrm{mg} / \mathrm{kg}$ causou a morte em quatro de sete bovinos inoculados. Lago et al. (2000) também causaram a morte em bovinos utilizando a dose de $0,03 \mathrm{mg} / \mathrm{kg}$ do veneno crotálico.

\section{Resultados laboratoriais}

No leucograma, tanto dos búfalos quanto dos bovinos, verificou-se leucocitose por neutrofilia. Graça et al. (2008) verificaram no leucograma de bovinos inoculados por $C$. durissus terrificus leucocitose por neutrofilia, além de linfopenia, eosinopenia e monocitose. Lago et al. (2001) observaram leucocitose por neutrofilia e linfocitose. A leucocitose por neutrofilia observada nesse estudo pode ter sido causada pelo estresse, determinado pela migração do "pool" marginal para o meio circulante, induzida pelo aumento da liberação de cortisol endógeno (Jain 1986).

A elevação nos níveis de CK também foi verificada por Lago et al. (2004) e Graça et al. (2008). Contudo, de acordo com Smith (2006) as análises laboratoriais de animais submetidos ao decúbito prolongado indicam elevação da atividade sérica de $\mathrm{CK}$ e, consequentemente, das atividades séricas de AST e DHL. Radostits et al. (2000) descrevem a síndrome da vaca caída com aumento dos níveis de CK. Graça et al. (2006) apud Graça et al. (2008) submeteram bovinos ao decúbito experimental por seis horas e verificaram, 18 horas após o início do experimento, aumento nos níveis de CK.

Portanto, além da ação miotóxica do veneno, os animais do experimento foram submetidos à contenção mecânica, punções venosas para coleta de sangue e decúbito prolongado, os estudos ligados ao decúbito permanente em bovinos indicam que os valores obtidos no presente experimento não sugerem um quadro acentuado de necrose muscular. Segundo Smith (2006), os limites normais da fosfatase alcalina podem estar aumentados em animais jovens, como é o caso dos animais utilizados no presente experimento.

\section{Achados de necropsia e histopatológicos}

No presente trabalho não se verificou alterações macroscópicas que pudessem caracterizar o diagnóstico do envenenamento crotálico em búfalos e em bovinos. 0 edema quase imperceptível observado em um dos bovinos, provavelmente passaria despercebido ao exame clínico e à necropsia; este achado também foi verificado por Graça et al. (2008). No bubalino necropsiado esta alteração não foi observada.

\section{Diagnóstico diferencial}

Segundo Tokarnia et al. (2002), os muitos casos de morte súbita na região Norte, geralmente são atribuídos as "picadas-de-cobra", superestimando a sua ocorrência, já que milhares de cabeças morrem anualmente intoxicados por Palicourea marcgravii. Por outro lado, já se sabe que $C$. durissus terrificus ocorre somente em algumas áreas da região Norte, estendendo-se por Rondônia, Amazonas e Pará (campos abertos de Humaitá, Serra do Cachimbo e Santarém) (Melgarejo 2009). Portanto, a importância da realização do diagnóstico diferencial com plantas que causam morte súbita, mas também com enfermidades do sistema nervoso central e periférico, principalmente botulismo, já que essa enfermidade pouco tem sido descrita em búfalos. Relatos de Langenegger \& Döbereiner (1988) mostram sintomatologia nervosa e nenhum achado significativo na macroscopia de búfalos com botulismo, achados semelhantes ao encontrado no presente trabalho.

\section{REFERÊNCIAS}

Amaral C.F.S., Magalhães R.A. \& Resende N.A. 1991. Comprometimento respiratório secundário a acidente ofídico crotálico. Revta Inst. Med. Trop. 33(4):251-255.

Araújo P., Rosenfeld G. \& Belluomini H.E. 1963. Toxicidade de venenos ofídicos. II. Doses mortais para bovinos. Arqs Inst. Biológico, São Paulo, 30:43-48.

Azevedo-Marques M.M., Hering S.E. \& Cupo P. 2009. Acidente crotálico, p.108-115. In: Cardoso J.L.C., França F.O.S., Wen F.H., Málaque C.M.S. \& Haddad Jr V. (Eds), Animais Peçonhentos no Brasil: Biologia, clínica e terapêutica dos acidentes. Sarvier Editora, São Paulo.

Belluomini H.E. 1972. Ensaios soroterápicos no envenenamento crotálico experimental em bovinos. Tese de Doutorado em Ciências Biomédicas, Universidade de São Paulo, São Paulo. 190p.

Belluomini H.E., Araujo P., Rosenfeld G., Leinz F.F. \& Birgel E.H. 1982. Symptomatologie der experimentellen Crotalustoxin-Vergiftung bei Rindern, die einer spezifischen Serumtherapie unterworfen wurden. Dtsch. Tierärztl. Wochenschr. 89(11):444-448.

Bicudo P.L. 1999. Acidentes ofídicos em Medicina Veterinária, p.375-387. In: Barraviera B. (Ed.), Venenos: aspectos clínicos e terapêuticos dos acidentes por animais peçonhentos. EPUB, Rio de Janeiro.

Graça F.A.S., Peixoto P.V., Coelho C.D., Caldas S.A. \& Tokarnia C.H. 2008. Aspectos clínico-patológicos e laboratoriais do envenenamento crotálico experimental em bovinos. Pesq. Vet. Bras. 28(6):261-270.

Jain N.C. 1986. Schalm's Veterinary Hematology. $4^{\text {th }}$ ed. Lea and Febiger, Philadelphia. 1221p.

Lago L.A. 1996. Avaliação clínica e laboratorial de bovinos submetidos ao envenenamento crotálico experimental (Crotalus durissus terrificus Laurenti, 1768) crotamina positivo. Dissertação de Mestrado em Medicina Veterinária, Universidade Federal de Minas Gerais, Belo Horizonte. 86p.

Lago L.A., Ferreira P.M., Facury Filho E.J., Melo M.M., Alzamora Filho F. 2000. Quadro clínico do envenenamento crotálico experimental em bo- 
vinos (Crotalus durissus terrificus - crotamina positivo). Braz. J. Vet. Res. Anim. Sci. 37(4):312-315.

Lago L.A., Melo M.M., Ferreira P.M. \& Facury Filho E.J. 2001. Alterações hematológicas em bovinos submetidos ao envenenamento crotálico. Revta Bras. Saúde Prod. Anim. 1(1):7-13.

Lago L.A., Marques Junior A.P., Melo M.M., Lago E.P., Oliveira N.J.F. \& Alzamora Filho F. 2004. Perfil bioquímico sorológico de bovinos inoculados experimentalmente com veneno crotálico iodado livre e iodado incorporado em liposomas. Arq. Bras. Med. Vet. Zootec. 56(5):653-657.

Langenegger J. \& Döbereiner J. 1988. Botulismo enzoótico em búfalos no Maranhão. Pesq. Vet. Bras. 8(1/2):37-42.

Melgarejo A.R. 2009. Serpentes peçonhentas do Brasil, p.42-69. In: Cardoso J.L.C., França F.O.S., Wen F.H., Málaque C.M.S. \& Haddad Jr V. (Eds), Animais Peçonhentos no Brasil: Biologia, clínica e terapêutica dos acidentes. Sarvier Editora, São Paulo.
Radostits E.M., Gay C.C., Blood D.C. \& Hinchcliff K.W. 2000. Veterinary Medicine. $9^{\text {th }}$ ed. W.B. Saunders, New York. 1877p.

Sisson S. 1986. Tegumento comum, p.1131-1134. In: Getty R. (Ed.), Anatomia dos Animais Domésticos. 5a ed. Guanabara Koogan, Rio de Janeiro.

Smith B.P. 2006. Medicina interna de grandes animais. $3^{\text {a }}$ ed. Manole, Barueri. 1728 p.

Sociedade Brasileira de Herpetologia. Lista brasileira de anfíbios e répteis. Disponível em <http://www.sbhepelologia.org.br/> Acesso em 28 abr. 2011.

Tokarnia C.H. \& Peixoto P.V. 2006. A importância dos acidentes ofídicos como causa de mortes em bovinos no Brasil. Pesq. Vet. Bras. 26(2):5568.

Tokarnia C.H., Döbereiner J. \& Peixoto P.V. 2002. Poisoning plants affecting livestock in Brazil. Toxicon 40(12):1635-1660. 